\title{
Factores asociados a la hospitalización de pacientes con COVID-19 en la Unidad de Cuidados Intensivos de una clínica en 2020
}

Ysabel Lozano* 1,a; Enver V. Palacios ${ }^{1, a}$

\section{RESUMEN}

Objetivo: Identificar los factores asociados a la hospitalización de los pacientes con COVID-19 en una unidad de cuidados intensivos.

Materiales y métodos: Estudio observacional, transversal y analítico. La muestra estuvo conformada por los pacientes con COVID-19 atendidos en la Clínica Good Hope durante el periodo de abril a junio de 2020. Las variables consideradas fueron las siguientes: variable resultado (el lugar de hospitalización: unidad de cuidados intensivos o servicio de medicina) y variable exposición (edad, sexo, tiempo de enfermedad, comorbilidades y exámenes de laboratorio como deshidrogenasa láctica, ferritina, dímero $\mathrm{D}$, proteína C-reactiva). Se realizó un análisis descriptivo, bivariado y regresión GLM Poisson simple para calcular razón de probabilidades (PR).

Resultados: Se evaluaron a 76 pacientes (60 en el servicio de medicina y 16 en la unidad de cuidados intensivos). La edad media fue 52 años y el sexo predominante fue el masculino (81,60\%). Los niveles séricos elevados de deshidrogenasa láctica, dímero $\mathrm{D}$ y proteína $\mathrm{C}$-reactiva se asociaron con el aumento de la probabilidad de ingreso en la unidad de cuidados intensivos al momento de la hospitalización en 0,02 \%, lo que es estadísticamente significativo (RP: 1,002; IC95\% 1,0011,003; RP: 1,05; IC95\% 1,01-1,10 y RP: 1,06; IC95\% 1,02-1,09).

Conclusiones: Los niveles de deshidrogenasa láctica, proteína C-reactiva y dímero $\mathrm{D}$ están asociados con el ingreso a una unidad de cuidados intensivos durante la hospitalización y podrían reflejar la severidad de la enfermedad.

Palabras clave: Biomarcadores; Inflamación; COVID-19 (Fuente: DeCS BIREME).

\section{Factors associated with the hospitalization of COVID-19 patients in a clinic's intensive care unit in 2020}

\section{ABSTRACT}

Objective: To identify the factors associated with the hospitalization of COVID-19 patients in an intensive care unit. Materials and methods: An observational, cross-sectional and analytical study. The sample consisted of COVID-19 patients treated at the Clínica Good Hope from April to June 2020. The variables were: outcome (place of hospitalization: intensive care unit or internal medicine service) and exposure (age, sex, disease period, comorbidities and laboratory tests such as levels of lactate dehydrogenase, ferritin, D-dimer and C-reactive protein). A descriptive, bivariate analysis and a simple GLM Poisson regression were performed to calculate the odds ratio (OR).

Results: Seventy-six (76) patients were evaluated (60 in the internal medicine service and 16 in the intensive care unit). The mean age was 52 years and males prevailed $(81.6 \%)$. Elevated serum levels of lactate dehydrogenase, D-dimer and $\mathrm{C}$-reactive protein were associated with a $0.02 \%$ more chance of admission to the intensive care unit at the time of hospitalization, which is statistically significant (OR: 1.002, 95 \% Cl: 1.001 - 1.003; OR: 1.05, 95 \% Cl: 1.01 - 1.10 ; and OR: 1.06, 95 \% Cl: 1.02 - 1.09).

Conclusions: The levels of lactate dehydrogenase, D-dimer and C-reactive protein are associated with the admission to an intensive care unit during hospitalization and could reflect the severity of the disease.

Keywords: Biomarkers; Inflammation; Coronavirus infections (Source: MeSH NLM).

1 Universidad Científica del Sur. Lima, Perú.

a Médico especialista en Medicina Interna.

*Autor corresponsal 


\section{INTRODUCCIÓN}

A finales de 2019, China fue el epicentro del brote de neumonía causado por un nuevo coronavirus (2019-nCoV), clasificado como SARS-CoV-2, causante de la enfermedad COVID-19. El 11 de marzo del 2020 la Organización Mundial de Salud (OMS) declaró a esta enfermedad como una pandemia. En el Perú, el primer caso de COVID-19 se identificó el 6 de marzo de 2020 en Lima, procedente de Europa. En la actualidad (21 de junio de 2020), el país registra 254936 casos confirmados, de los cuales 141967 $(55,69 \%)$ han sido dados de alta y $8045(3,16 \%)$ fallecieron. Lima es la región con mayor cantidad de infectados y el principal foco de la pandemia, seguida de las regiones Callao y Piura ${ }^{(1)}$. La tasa de contagio por cada 100000 habitantes es de $0,87 \%$ a nivel nacional. El Callao ha sido la región que concentró la mayor tasa de infección $(1,71 \%)$, seguido por Lima $(1,52 \%)$ y Ucayali $(1,41 \%)$. En cuanto a la tasa de letalidad, Ica ocupa el primer lugar con un valor registrado de 6,62 \%, seguida de Ancash y Lambayeque (2). El sistema de salud se ve desbordado en su capacidad por la carencia de camas en las unidades de cuidados intensivos ( $\mathrm{UCl}$ ) y en hospitalización; además de otros recursos necesarios en la atención de estos pacientes, lo que constituye una dura prueba para nuestro sistema de salud.

Los síntomas más frecuentes de la enfermedad son fiebre, dolor de garganta, tos y disnea. El $80 \%$ de los casos presenta formas leves que se autolimitan; sin embargo, el $15 \%$ se manifiesta de forma severa o grave y requiere manejo intrahospitalario ${ }^{(3,4)}$ por un gran proceso inflamatorio que ocasiona una disfunción multiorgánica asociada a una alta mortalidad. Frente a ello se debe optimizar la atención de los pacientes, identificando tempranamente a aquellos con riesgo de progresar a cuadros severos o graves mediante una evaluación clínica completa que investigue las comorbilidades y los marcadores inflamatorios. Diversos estudios han evaluado los factores relacionados a mal pronóstico y a las formas severas de la enfermedad. Se han identificado varios parámetros clínicos, como edad avanzada, comorbilidades (obesidad, hipertensión arterial, diabetes mellitus), y también parámetros de laboratorio, por ejemplo, marcadores hematológicos (leucocitos, neutrófilos, dímero D), inflamatorios (ferritina, proteína C-reactiva o PCR, entre otros) y cardiacos. Estos datos son importantes para identificar de manera precoz a los pacientes con riesgo a avanzar a cuadros graves de la enfermedad y a la muerte ${ }^{(5-9)}$. De esta manera se podrá optimizar el uso de los recursos sanitarios. La crisis desatada por la aparición de una nueva enfermedad, la COVID-19, constituye una dura prueba para el sistema de salud de nuestro país, dada la carencia de camas hospitalarias en las unidades de cuidados intensivos ( $\mathrm{UCI}$ ), además de otros recursos necesarios en la atención de estos pacientes.

Debido a que, en el contexto de la pandemia, los estudios se han realizado en poblaciones distintas a la nuestra, en países de primer mundo con un perfil epidemiológico diferente, el presente estudio tiene por finalidad identificar los factores asociados a la hospitalización de los pacientes con COVID-19 en una UCl para poder identificarlos tempranamente y, así, brindarles una atención oportuna.

\section{MATERIALES Y MÉTODOS}

\section{Diseño y población de estudio}

Se realizó un estudio observacional, y transversal analítico. La población estuvo constituida por 372 pacientes con diagnóstico de COVID-19 atendidos en el Servicio de Medicina de Emergencia de la clínica Good Hope durante el periodo de mayo-junio 2020. De estos, se seleccionaron 76 historias clínicas completas y las demás no se incluyeron por estar incompletas o no cumplir criterios de elegibilidad. Estos 76 pacientes fueron clasificados en dos grupos dependiendo de si eran hospitalizados en el Departamento de Medicina o en la Unidad de Cuidados Intensivos (UCI) de la clínica, de acuerdo a la evaluación y decisión del médico de guardia. Se realizó un muestreo no probabilístico, por conveniencia, de las historias clínicas de pacientes a partir del listado de las mismas según código $\mathrm{CIE} 10$ obtenido de los registros electrónicos de la institución. Se consideró como caso a todo paciente mayor de 18 años con diagnóstico de COVID-19 realizado mediante signos clínicos, radiológicos y prueba molecular y/o prueba rápida para COVID-19. Se excluyeron a los que presentaran datos incompletos de la historia clínica y a las gestantes.

\section{Variables y mediciones}

Las variables consideradas en el estudio fueron las siguientes: la variable resultado (lugar de hospitalización: área donde se hospitaliza el paciente, que puede ser servicio de medicina o $\mathrm{UCl}$ ), que fue manejada como una variable cualitativa dicotómica con valores 1 para $\mathrm{UCl}$ y 0 para Medicina; y la variable exposición, que incluye edad, sexo, tiempo de enfermedad, comorbilidades y exámenes de laboratorio tomados al momento de su ingreso al servicio de emergencia, como deshidrogenasa láctica (DHL), ferritina, dímero D y PCR. La edad, tiempo de enfermedad y los exámenes de laboratorio se analizaron como variables continuas.

\section{Análisis estadístico}

El paquete Stata 15 para Windows fue empleado para el análisis estadístico. Primero, se realizó un análisis descriptivo para evaluar las características basales de la población de estudio. Los datos cualitativos se presentan en forma de frecuencias absolutas y porcentajes, y los datos cuantitativos mediante las medidas de tendencia central y de dispersión, como media, mediana, desviación estándar (DS) y rango interquartilo (RIQ). Luego se dividió a la muestra en dos grupos: hospitalizados en $\mathrm{UCl}$ y en medicina mediante un análisis bivariado entre los dos 
grupos: las variables cualitativas (sexo y comorbilidades) se analizaron mediante el test de $\mathrm{X}^{2} \mathrm{o}$ el test exacto de Fisher, y las cuantitativas (edad, exámenes de laboratorio, tiempo de enfermedad) con la prueba t de Student o con técnicas no paramétricas, de acuerdo a la distribución de la variable. Finalmente, se realizó un modelo de regresión GLM poisson log simple para el cálculo de la razón de prevalencia y sus respectivos intervalos de confianza. Se consideró como nivel de significancia estadística $p<0,05$.

\section{Aspectos éticos}

Para la realización del presente estudio no se tuvo contacto alguno con humanos. En tal sentido, los posibles riesgos para los sujetos del análisis son mínimos y están relacionados, principalmente, a una brecha en la confidencialidad, la cual fue manejada mediante la codificación de historias clínicas. El protocolo fue evaluado y aprobado por el Comité de Ética de la institución. Durante la implementación del estudio se respetaron los principios éticos delineados en la Declaración de Helsinki.

\section{RESULTADOS}

Evaluamos a 76 pacientes. Luego de ser examinados en el Servicio de Emergencia, se agruparon 60 hospitalizados en Medicina y 16 en UCl. La media de edad fue de 52 años $(D S \pm 13)$ y predominó el sexo masculino $(81,58 \%)$. Los pacientes acudieron con una media de tiempo de enfermedad de 9 días $\left(\mathrm{DS}_{ \pm} 4\right)$ y reportaron síntomas como fiebre, tos y disnea. Asimismo, debemos destacar que presentaron una baja frecuencia de comorbilidades, como diabetes mellitus (21\%), hipertensión arterial (14\%), obesidad $(5,3 \%)$ y asma $(3,9 \%)$ (Tabla1).

Tabla 1. Características de los pacientes hospitalizados con COVID-19. Lima-Perú, 2020

Características

$N(\%)$

\begin{tabular}{|c|c|c|}
\hline Edad (años)* & & $52 \pm 13$ \\
\hline \multirow[t]{2}{*}{ Sexo } & Masculino & $62(81,58)$ \\
\hline & Femenino & $14(18,42)$ \\
\hline \multicolumn{3}{|l|}{ Síntomas } \\
\hline & Fiebre & $60(80,00)$ \\
\hline & Dolor de garganta & $23(30,26)$ \\
\hline & Tos & $57(76,00)$ \\
\hline & Disnea & $69(92,00)$ \\
\hline \multicolumn{3}{|l|}{ Comorbilidades } \\
\hline & Hipertensión arterial & $11(14,47)$ \\
\hline & Diabetes mellitus & $16(21,05)$ \\
\hline & Asma & $4(5,26)$ \\
\hline & Obesidad & $3(3,95)$ \\
\hline & & $10361 \pm 4478$ \\
\hline Leucocitos $(\mathrm{cel} / \mu \mathrm{l})$ * & & $1091 \pm 465$ \\
\hline Linfocitos $(\mathrm{cel} / \mu \mathrm{l})$ * & & $287335 \pm 122800$ \\
\hline Plaquetas $(\mathrm{cel} / \mu \mathrm{l})$ * & & $0,80 \pm 0,23$ \\
\hline Creatinina $(\mathrm{mg} / \mathrm{dl})$ * & & $591 \pm 263$ \\
\hline Deshidrogenasa láctica (UI/L) * & & $17,8 \pm 10,9$ \\
\hline Proteína C-reactiva $(\mathrm{mg} / \mathrm{dl})$ * & & $0,79[0,10-34,7]$ \\
\hline Dímero D (mg/L) † & & $1305[38,50-6531]$ \\
\hline \multicolumn{3}{|l|}{ Ferritina(ng/ml) $\dagger$} \\
\hline Tiempo de enfermedad (días)* & & $9,5 \pm 4,2$ \\
\hline
\end{tabular}




\begin{tabular}{lll}
$\begin{array}{l}\text { Características } \\
\text { Lugar de hospitalización }\end{array}$ & N $(\%)$ \\
\hline & Medicina & $60(78,95)$ \\
\hline & UCl & $16(21,05)$ \\
\hline & Categoría & \\
\hline * Media \pm desviación estándar & & \\
† Mediana [Rango interquartilo] &
\end{tabular}

En la tabla 2 se muestra que los pacientes con COVID-19 que se hospitalizaron en UCI fueron, en su mayoría, varones y de mayor edad comparados con los hospitalizados en Medicina (93,75 \% vs. $78,33 \%$; 57 años vs. 51 años). Sin embargo, esta diferencia no fue estadísticamente significativa $p=0,277$ y $p=0,15$. No se evidenció diferencia en la presencia de las comorbilidades anteriormente referidas.

Tabla 2. Factores asociados al ingreso a UCI en pacientes con COVID-19. Clínica Good Hope. Lima-Perú, 2020

Variables

Lugar de hospitalización

Medicina $(n=60)$

N (\%)
UCI $(n=16)$

N (\%)

Sexo

$13(21,67)$

$47(78,33)$ $p$

0,28

$1(6,25)$

$15(93,75)$

Femenino

Masculino

\section{Comorbilidades}

\begin{tabular}{|c|c|c|c|c|}
\hline & Hipertensión arterial & $8(13,33)$ & $3(18,75)$ & 0,58 \\
\hline & Diabetes mellitus & $11(18,33)$ & $5(31,25)$ & 0,26 \\
\hline & Obesidad & $3(5,00)$ & $0(0,0)$ & 1 \\
\hline & Asma & $4(6,67)$ & $0(0,0)$ & 0,57 \\
\hline \multicolumn{2}{|c|}{ Edad (años)* } & $51 \pm 1,7$ & $57 \pm 3,8$ & 0,15 \\
\hline \multicolumn{2}{|c|}{ Tiempo de enfermedad (días) $\dagger$} & $10[7-14]$ & $7,5[5,5-13,5]$ & 0,36 \\
\hline \multicolumn{5}{|c|}{ Exámenes auxiliares } \\
\hline . & Leucocitos $(\mathrm{cel} / \mu \mathrm{l}) \dagger$ & 9345 [6790-12 525] & $9780[8665-10780]$ & 0,77 \\
\hline . & Linfocitos (cel/ $\mu \dagger$ & 1075 [805-1365] & 955 [665-1095] & 0,19 \\
\hline - & Urea $(\mathrm{mg} / \mathrm{dl}) \dagger$ & $32,1[24,40-36,10]$ & $41,2[25,90-51,40]$ & 0,14 \\
\hline . & Creatinina $(\mathrm{mg} / \mathrm{dl}) \dagger$ & $0,81[0,68-0,91]$ & $0,72[0,62-0,86]$ & 0,25 \\
\hline . & Deshidrogenasa láctica (UI/L) † & $487[395,5-683]$ & $832[706,5-967,5]$ & $<0,001$ \\
\hline
\end{tabular}




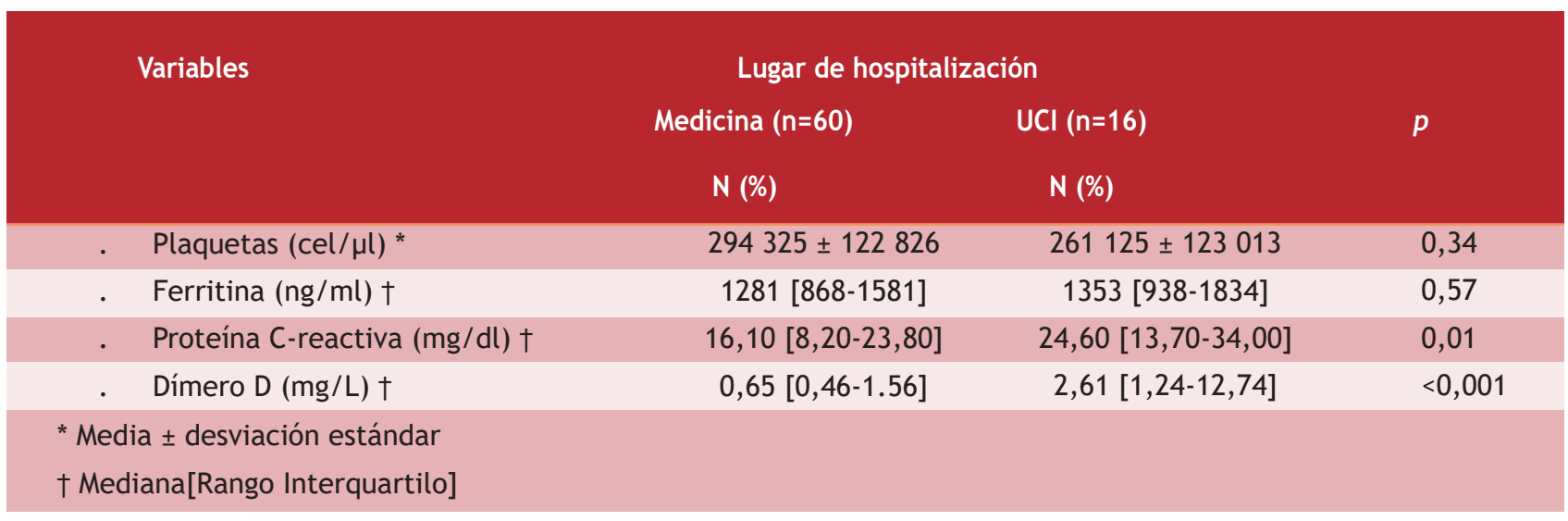

En relación a los exámenes auxiliares, los pacientes hospitalizados en UCI mostraron valores mayores de DHL, PCR y dímero $D$ ( 832 vs. $487 ; 24,6$ vs. 16,1 y 2,61 vs. 0,65 , respectivamente) que son estadísticamente significativos $(p \leq 0,01)$ (Figuras 1,2 y 3 ).

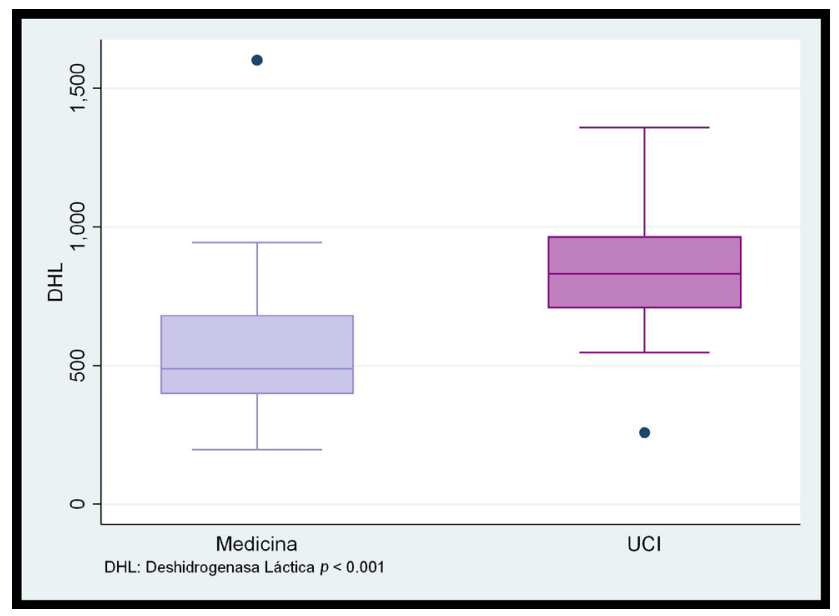

Figura 1. Niveles séricos de DHL en pacientes con COVID-19, según lugar de hospitalización. Clínica Good Hope. Lima, Perú 2020

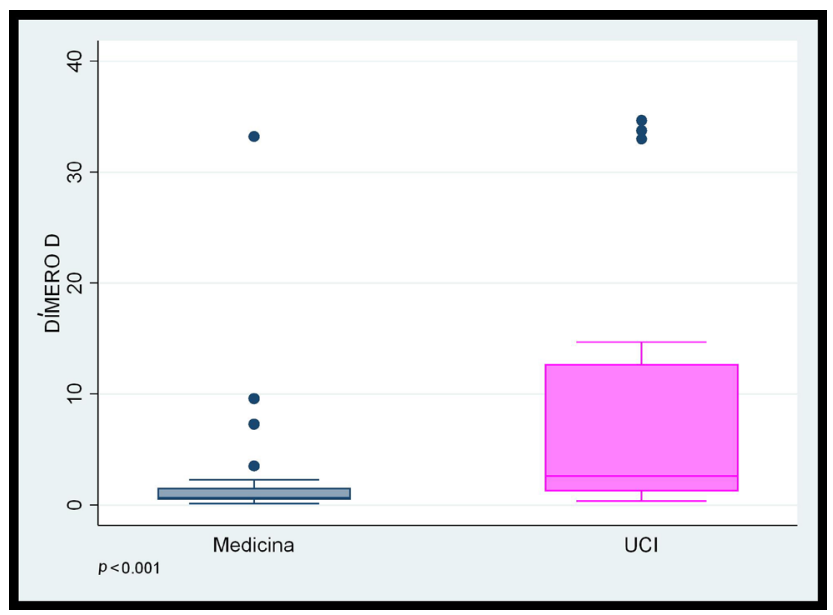

Figura 2. Niveles séricos de dímero D en pacientes con COVID-19, según lugar de hospitalización. Clínica Good Hope. Lima, Perú 2020 


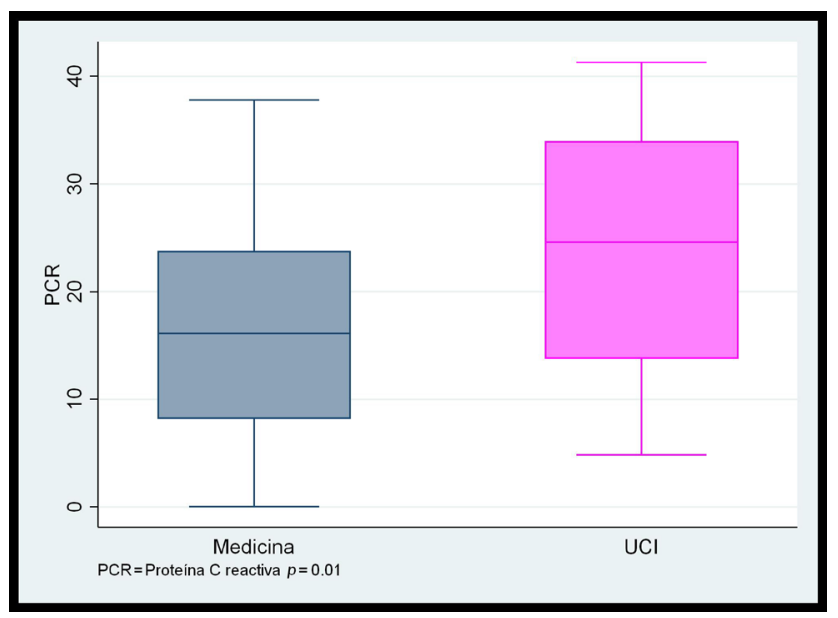

Figura 3. Niveles séricos de proteína C-reactiva en pacientes con COVID-19, según lugar de hospitalización. Clínica Good Hope. Lima, Perú 2020

La regresión GLM simple muestra que el incremento de los niveles séricos de DHL, PCR y dímero $D$ se asociaron al aumento de la probabilidad de ingreso a la $\mathrm{UCl}$ al momento de la hospitalización, y que fueron estadísticamente significativos (RP: 1.002, IC95 \% 1,001-1,003; RP: 1,05, IC95 \% 1,01-1,10 y RP: 1,06 IC95 \% 1,02-1,09). Asimismo, se observó que el incremento de la edad aumenta la probabilidad de ingreso a $\mathrm{UCl}$ en $2,00 \%$, pero no fue estadísticamente significativo. De otro lado, se encontró una tendencia a mayor probabilidad de ingreso a $\mathrm{UCl}$ cuando el enfermo es del sexo masculino (RP: $3,39, p=0,238$ ). No se encontró asociación entre la presencia de comorbilidades y la probabilidad de ingreso a UCI (Tabla 3).

Tabla 3. Factores asociados a la hospitalización en UCI de los pacientes COVID-19 en análisis de regresión simple. Clínica Good Hope. Lima- Perú, 2020

\begin{tabular}{|c|c|c|c|}
\hline \multirow[t]{2}{*}{ Características } & \multicolumn{3}{|c|}{ Análisis bivariado } \\
\hline & RP & IC $95 \%$ & $p$ \\
\hline Edad & 1,02 & $0,98-1,06$ & 0,20 \\
\hline Sexo & & & 0.24 \\
\hline Femenino & Ref. & & \\
\hline Masculino & 3,39 & $0,45-25,64$ & \\
\hline Diabetes mellitus & & & 0.99 \\
\hline No & Ref. & & \\
\hline Sí & 1,70 & $0,59-4,91$ & \\
\hline Hipertensión arterial & & & 0,63 \\
\hline No & Ref. & & \\
\hline Sí & 1,36 & $0,39-4,79$ & \\
\hline Deshidrogenasa láctica & 1,002 & $1,001-1,003$ & $<0,01$ \\
\hline
\end{tabular}




\begin{tabular}{|c|c|c|c|}
\hline \multirow[t]{2}{*}{ Características } & \multicolumn{3}{|c|}{ Análisis bivariado } \\
\hline & RP & IC $95 \%$ & $p$ \\
\hline Dímero D & 1,06 & $1,02-1,09$ & $<0,01$ \\
\hline Proteína C-reactiva & 1.05 & $1.01-1.10$ & 0.03 \\
\hline Ferritina & 1 & $0,999-1$ & 0,84 \\
\hline
\end{tabular}

\section{DISCUSIÓN}

El presente estudio evaluó a 76 pacientes con COVID-19, de los cuales 60 fueron hospitalizados en Medicina y 16, en $\mathrm{UCl}$. Los niveles séricos de DHL, dímero $\mathrm{D}$ y PCR al ingreso estaban elevados, lo cual se asocia a una mayor probabilidad de hospitalización en UCI (RP: 1,002, IC95\% 1,001-1,003; RP: 1,06, IC95\% 1,02-1,09; RP: 1,05, IC95\% $1,01-1,10$; respectivamente).

La DHL es una enzima que se encuentra en diversas células del cuerpo, y tiene una mayor concentración en el corazón, hígado, músculos, riñones y células sanguíneas; es considerada como un indicador de daño agudo o crónico y marcador inflamatorio. En relación a los niveles altos de DHL y lugar de hospitalización, el estudio transversal de Fan et al. (10) evidenció que un valor elevado de esta enzima es un marcador de severidad y mal pronóstico en los pacientes con COVID-19 ${ }^{(11,12)}$ cuando se asocia con otros parámetros inflamatorios como el dímero $D$ y PCR, que son elementos importantes al momento de estratificar riesgo de progresión de una enfermedad o presentación de sus formas severas (11). Poggiali et al. (13) encontraron una correlación moderada inversa entre los niveles de $\mathrm{DHL}$ y los valores de $\mathrm{PaO}_{2} / \mathrm{FiO}_{2}$, lo que podría ser un importante predictor de falla respiratoria en pacientes con COVID-19.

En relación al dímero $D$, diversas investigaciones (11,14-17) han demostrado una relación entre cantidades elevadas, severidad del cuadro y necesidad de terapia en UCI. En un estudio ${ }^{(14)}$, los valores de dímero $D$ mayores de $2 \mathrm{ugr} / \mathrm{mL}$ se asociaron a valores elevados de PCR y linfopenia, por lo que se comporta como un predictor precoz de mortalidad en los pacientes que requirieron hospitalización. Gao et al. (17) evaluaron diversos parámetros de laboratorio en el diagnóstico diferencial de neumonía severa y encontraron, respecto al dímero $\mathrm{D}$, un área bajo la curva de 0,750 ( $p=0,0053)$, con un nivel crítico $0,28 \mathrm{ng} / \mathrm{L}$. Un metaanálisis de Huang et al. halló una asociación entre los niveles de dímero $D$ y el incremento de mortalidad (RR 4, 15 [2,43; 7,08], $p<0,001 ; 12: 83 \%$, $p=0,01)$, COVID-19 severo (RR $2,42[1,72 ; 3,40), p<$
$0,001 ; 12: 58 \%, p=0,05)$, mas no con la necesidad de tratamiento en UCI (RR 0,94 [0,43, 2,07], $p=0,88)$. Sin embargo, se debe señalar que los estudios tuvieron una elevada heterogeneidad. La elevación del dímero D indicaría un estado de hipercoagulabilidad en el paciente con COVID-19, esto explicaría por qué la infección viral promueve una respuesta proinflamatoria agresiva con un insuficiente control de la respuesta antiinflamatoria, lo que induciría una disfunción de las células endoteliales y ocasionaría una producción excesiva de trombina. De otro lado, la hipoxia, encontrada en los pacientes con COVID-19 severa, y el estado de postración incrementarían el riesgo de trombosis.

Con respecto a la PCR, diversos trabajos $(11-13,15,18-20)$ han evidenciado una asociación positiva entre la severidad de la enfermedad y los niveles de PCR, lo que constituye un posible factor pronóstico de severidad y progresión de enfermedad, inclusive en etapas tempranas. En un estudio retrospectivo, Tan et al. (21) encontraron una correlación entre la PCR y la severidad tomográfica de la enfermedad, así como una mayor estancia hospitalaria. Sahu et al., en un metanálisis (22), también evidenciaron esta asociación al mostrar que los pacientes fallecidos por COVID-19 tuvieron niveles séricos más elevados de PCR que los que no murieron ( $p=0,000$, diferencia standard entre las medias $=1,37)$. Asimismo, Huang et al. hallaron que los niveles de PCR se asociaron a mala evolución y aparición de formas severas de la enfermedad (RR 1,41 $[1,14 ; 1,74], p=0,002 ; 12: 93 \%, p<0,001)$ y necesidad de manejo en UCI (RR 1,96 [1,40; 2,74], $p<0,001)$. Herold et al. ${ }^{(23)}$ evidenciaron que los niveles de PCR predicen la necesidad de ingresar a ventilación mecánica con un área bajo la curva de 0,86 y 0,83 y un punto de corte de $97 \mathrm{mg} / \mathrm{L}$ para lograr una correcta clasificación, en un $80 \%$ de los pacientes. En tanto, para Poggiali et al. ${ }^{(13)}$ existe una correlación inversa moderada entre los niveles de PCR y los valores de $\mathrm{PaO}_{2} / \mathrm{FiO}_{2}\left(r=0,55 ; r^{2} 0,31, p<0,0001\right)$; además, en la regresión logística múltiple se evidenció que valores de $\mathrm{PaO}_{2} / \mathrm{FiO}_{2}$ menores de $200 \mathrm{mmHg}$ se asociaron significativamente con valores más elevados de DHL y PCR $(p<0,05)$. 
Las formas severas y graves de la enfermedad implican un estado de hiperinflamación con compromiso multiorgánico debido a la tormenta de citocinas propuesta por Siddiqi y Mehra (24), que encuentran múltiples marcadores inflamatorios elevados, entre ellos la ferritina, aunque en nuestra investigación no observamos diferencias entre el grupo hospitalizado en $\mathrm{UCl}$ y el de Medicina. Diversos estudios han encontrado relación entre los niveles de ferritina, complicaciones, severidad, mortalidad y necesidad de $\mathrm{UCl}$, tal como lo evidenciaron Huang et al. ${ }^{(15)}$, quienes encontraron niveles más elevados de ferritina en los pacientes que tuvieron peor evolución ( $p<0,0001 ; 12: 76 \%)$, aquellos que fallecieron (diferencia media estandarizada o SMD $0,96[0,78 ; 1,13], p<0,00001 ; 12: 0 \%, p=0,41)$ y los que tuvieron COVID 19 severo (SMD 0,97 [0,43; $1,50], p<0,004 ; 12: 82 \%, p=0,001$ ); sin embargo, la heterogeneidad de los estudios fue alta.

En cuanto a la edad, en nuestro estudio la población tuvo una media de 52 años; no encontramos diferencias entre el grupo que se hospitalizó en UCl y el de Medicina. En dos series de pacientes asiáticos ${ }^{(8,25)}$ se observó una mayor prevalencia de la enfermedad en varones $(62-73 \%$ ) y una mediana de edad de 49 (IQR, 41-58) y 56 (IQR 46-67) años, resultados similares a nuestros hallazgos. Diversos estudios ${ }^{\left({ }^{(10)}\right.}$ evidencian que, a mayor edad, mayor riesgo de ser hospitalizado por COVID-19, debido a formas severas y complicaciones de la enfermedad. Este aumento de morbimortalidad con la edad está asociado a las comorbilidades, especialmente la enfermedad cardiovascular (ECV), y a la situación de susceptibilidad que conlleva una respuesta inmunológica más pobre. Wynants et al. ${ }^{(26)}$, en un metanálisis de 27 estudios, identificaron a la edad, el sexo masculino, los ingresos hospitalarios previos, comorbilidades y determinantes sociales de la salud como factores pronósticos asociados a la hospitalización en pacientes con COVID-19 mediante tres modelos predictivos, con un índice $C$ estimado de 0,$73 ; 0,81$ y 0,81 cada uno. Frente a esto, la edad ha sido considerada un factor pronóstico para presentar formas severas y mayor riesgo de ingreso a $\mathrm{UCl}$. En nuestro estudio no se evidenció esta asociación, lo cual podría estar relacionado con los criterios de ingreso a $\mathrm{UCl}$ implementados durante la pandemia en relación con la edad.

El mayor porcentaje de pacientes fue del sexo masculino $(81,60 \%)$. Aunque las personas de ambos sexos tienen el mismo riesgo de infectarse con el virus, se observa que los hombres son significativamente más propensos a presentar las formas severas de la enfermedad, requieren hospitalización e ingreso a $\mathrm{UCl}$, y en algunos casos, fallecen. Jin et al. ${ }^{(27)}$ evaluaron las diferencias de sexo en los pacientes con COVID-19, para lo cual analizaron una serie de casos de 43 pacientes hospitalizados, una base de datos pública de 1056 pacientes COVID-19 y una cohorte de
524 pacientes del brote de SARS de 2003, y hallaron, en el análisis de la serie de casos, que los hombres tuvieron un cuadro más severo que las mujeres $(p=0,035)$; en tanto que en la base de datos los varones tuvieron 2,4 veces más riesgo de morir que las mujeres (70,3 vs. $29,7 \%, p=0,016)$. Este comportamiento también se observó en la cohorte de SARS, en la que el grupo de fallecidos estuvo conformado por un mayor porcentaje de varones $(p=0,015)$.

Nuestro estudio evidencia una clara asociación entre los niveles elevados de DHL, dímero D y PCR y la probabilidad de ingreso a UCl. Como limitaciones del estudio debemos considerar que obtuvimos los datos a partir de los registros de las historias clínicas que, en algunos casos, no consignaron algunas variables como el índice de masa corporal, por lo que no registramos la asociación con este factor. Asimismo, debemos indicar que el tamaño de muestra es pequeño, lo cual podría tener impacto en los resultados y, finalmente, al ser un estudio retrospectivo, no se pudo tener una evaluación continua de los parámetros estudiados.

Por otro lado, es preciso señalar que la mayoría de los estudios que evalúan la asociación entre los parámetros clínicos y bioquímicos y la severidad de la COVID-19 son principalmente europeos, asiáticos y norteamericanos; la evidencia en los ámbitos latinoamericanos y nacional es escasa o nula, por lo que se debe plantear la posibilidad de realizar estudios en nuestra población para optimizar al máximo el uso de los escasos recursos con los que se cuenta en el sistema de salud y, de esta manera, poder brindar una rápida y oportuna atención a los pacientes que se encuentran en alto riesgo de complicaciones e, incluso, de morir.

Agradecimientos: A todos los médicos que día a día se enfrentan a esta pandemia con fortaleza, fe y esperanza.

Contribuciones de los autores: Ysabel Lozano y Enver V. Palacios realizaron la base de datos y el documento extenso del artículo.

Fuentes de financiamiento: Este artículo ha sido financiado por los autores.

Conflictos de interés: Los autores declaran no tener ningún conflicto de interés.

\section{REFERENCIAS BIBLIOGRÁFICAS}

1. Ministerio de Salud. Casos confirmados por Coronavirus Covid-19 ascienden a 244388 en el Perú (Comunicado $N^{\circ}$ 137) [Internet]. Lima; 2020. Disponible en: https://www.gob.pe/institucion/minsa/ noticias/187617-minsa-casos-confirmados-por-coronavirus-covid-19ascienden-a-244-388-en-el-peru-comunicado-n-137

2. Ministerio de Salud. Covid 19 en el Perú - Sala situacional [Internet]. Lima; 2020. Disponible en: https://covid19.minsa.gob.pe/sala_ situacional.asp

3. Shi Y, Wang G, Cai X-P, Deng J-W, Zheng L, Zhu H-H, et al. An 
overview of COVID-19. J Zhejiang Univ Sci B. 2020; 21: 343-60.

4. Wang D, Yin Y, Hu C, Liu X, Zhang X, Zhou S, et al. Clinical course and outcome of 107 patients infected with the novel coronavirus, SARSCoV-2, discharged from two hospitals in Wuhan, China. Crit Care. 2020; 24(1): 188.

5. Henry BM, Santos De Oliveira MH, Benoit S, Plebani M, Lippi G. Hematologic, biochemical and immune biomarker abnormalities associated with severe illness and mortality in coronavirus disease 2019 (COVID-19): a meta-analysis. Clin Chem Lab Med. 2020; 58(7): 1021-8.

6. Du R-H, Liang L-R, Yang C-Q, Wang W, Cao T-Z, Li M, et al. Predictors of mortality for patients with COVID-19 pneumonia caused by SARSCoV- 2: A prospective cohort study. Eur Respir J. 2020; 55(5): 2000524.

7. Ponti G, Maccaferri M, Ruini C, Tomasi A, Ozben T. Biomarkers associated with COVID-19 disease progression. Crit Rev Clin Lab Sci. 2020; 1-11.

8. Zhou F, Yu T, Du R, Fan G, Liu Y, Liu Z, et al. Clinical course and risk factors for mortality of adult inpatients with COVID-19 in Wuhan, China: a retrospective cohort study. Lancet. 2020; 395(10229): 1054-62.

9. Ahn D-G, Shin H-J, Kim M-H, Lee S, Kim H-S, Myoung J, et al. Current status of epidemiology, diagnosis, therapeutics, and vaccines for novel coronavirus disease 2019 (COVID-19). J Microbiol Biotechnol. 2020; 30(3): 313-24.

10. Fan BE, Lian Chong VC, Wei Chan SS, Lim GH, Eric Lim KG, Tan GB, et al. Hematologic parameters in patients with COVID-19 infection. Am J Hematol. 2020; 95(6): E131-4.

11. Velavan TP, Meyer CG. Mild versus severe COVID-19: Laboratory markers. Int J Infec Dis. 2020; 95: 304-7.

12. Lagadinou M, Salomou EE, Zareifopoulos N, Marangos M, Gogos C, Velissaris D. Prognosis of COVID-19: Changes in laboratory parameters. Le Infez Med. 2020; 28(Suppl. 1): 89-95.

13. Poggiali E, Zaino D, Immovilli P, Rovero L, Losi G, Dacrema A, et al. Lactate dehydrogenase and $\mathrm{C}$-reactive protein as predictors of respiratory failure in CoVID-19 patients. Clin Chim Acta. 2020; 509: $135-8$

14. Tang N, Li D, Wang X, Sun Z. Abnormal coagulation parameters are associated with poor prognosis in patients with novel coronavirus pneumonia. J Thromb Haemost. 2020; 18(4): 844-7.

15. Huang I, Pranata R, Lim MA, Oehadian A, Alisjahbana B. C-reactive protein, procalcitonin, D-dimer, and ferritin in severe coronavirus disease-2019: a meta-analysis. Ther Adv Respir Dis. 2020; 14: 175346662093717.

16. Yan D, Liu X-Y, Zhu Y, Huang L, Dan B, Zhang G, et al. Factors associated with prolonged viral shedding and impact of Lopinavir/ Ritonavir treatment in hospitalised non-critically ill patients with SARS-CoV-2 infection. Eur Respir J. 2020; 56(1): 2000799.

17. Gao Y, Li T, Han M, Li X, Wu D, Xu Y, et al. Diagnostic utility of clinical laboratory data determinations for patients with the severe COVID-19. J Med Virol. 2020; 92(7): 791-6.

18. Ruan Q, Yang K, Wang W, Jiang L, Song J. Clinical predictors of mortality due to COVID-19 based on an analysis of data of 150 patients from Wuhan, China. Intensive Care Med. 2020; 46(5): 846-8.

19. Wang L. C-reactive protein levels in the early stage of COVID-19. Med Mal Infect. 2020; 50(4): 332-4.

20. Gamboa-Acuña B, Guillén-Zambrano R, Lizzetti-Mendoza G, Soto A, Lucchetti-Rodríguez A. Factores asociados a sobrevida en pacientes con co-infección VIH-TBC en el Servicio de Infectología del Hospital Nacional Arzobispo Loayza , Perú, durante los años 2004-2012. Rev Chil Infect. 2018; 35(1): 41-8.

21. Tan C, Huang Y, Shi F, Tan K, Ma Q, Chen Y, et al. C-reactive protein correlates with computed tomographic findings and predicts severe COVID-19 early. J Med Virol. 2020; 92(7): 856-62.
22. Sahu BR, Kampa RK, Padhi A, Panda AK. C-reactive protein: A promising biomarker for poor prognosis in COVID-19 infection. Clin Chim Acta. 2020; 509: 91-4.

23. Herold T, Jurinovic V, Arnreich C, Lipworth BJ, Hellmuth JC, Bergwelt-Baildon MV, et al. Elevated levels of IL-6 and CRP predict the need for mechanical ventilation in COVID-19. J Allergy Clin Immunol. 2020; 146(1): 128-36.

24. Siddiqi HK, Mehra MR. COVID-19 illness in native and immunosuppressed states: A clinical-therapeutic staging proposal. $J$ Heart Lung Transplant. 2020; 39(5): 405-7.

25. Huang C, Wang Y, Li X, Ren L, Zhao J, Hu Y, et al. Clinical features of patients infected with 2019 novel coronavirus in Wuhan, China. Lancet. 2020; 395(10223): 497-506.

26. Wynants L, Van Calster B, Bonten MM, Collins G, Debray TP, De Vos $M$, et al. Systematic review and critical appraisal of prediction models for diagnosis and prognosis of COVID-19 infection. BMJ. 2020; 369: m1328.

27. Jin J-M, Bai P, He W, Wu F, Liu X-F, Han D-M, et al. Gender differences in patients With COVID-19: focus on severity and mortality. Front Public Health. 2020; 8: 152.

\section{Correspondencia:}

\section{Ysabel Lozano Rodas}

Dirección: Calle Los Sauces 285, urbanización La Fortaleza, San Juan de Miraflores. Lima, Perú.

Teléfono: 974636541

Correo electrónico: onisa2012@hotmail.com

Recibido: 16 de octubre de 2020

Evaluado: 11 de enero de 2021 Aprobado: 11 de febrero de 2021

(c) La revista. Publicado por Universidad de San Martín de Porres, Perú. (c) Br Licencia de Creative Commons Artículo en acceso abierto bajo términos de Licencia Creative Commons Atribución 4.0 Internacional. (http://creativecommons.org/licenses/by/4.0/)

ORCID iDs

Ysabel Lozano i https: / / orcid.org/0000-0002-9723-975X

Enver V. Palacios 1 https: / / orcid.org/0000-0002-0832-8751 